\title{
Harmonic Detection and Suppression of Frequency Doubling of Speed Measuring Radar Based on Closed-Loop Gain Control
}

\author{
Qingjiang Jin ${ }^{1,}$, Zongtian Liu ${ }^{1, b}$ and Qiuhong $\mathrm{Xu}^{2, \mathrm{c}}$ \\ ${ }^{1}$ School of Computer Engineering and Science Shanghai University, China \\ ${ }^{2}$ Hefei Vocation Science and Technology Co. Ltd

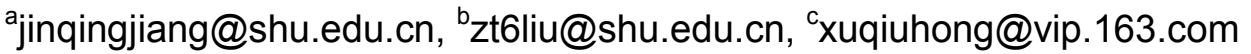

Keywords: Speed measuring, radar, harmonic detection, frequency doubling suppression

\begin{abstract}
This paper investigates the problem of frequency doubling with speed measuring radars. The main reason for it is that the gain of the reflected wave receiver cannot cope with all the possible waves reflected by cars of different distances away from the radar. Strong signals will saturate the receiver and generate harmonics which interferes with the speed measuring. Based on this, a harmonic detection and frequency doubling suppression method is developed in this paper and the effectiveness of the method is verified by experimental results.
\end{abstract}

\section{Introduction}

Frequency Doubling, as a general problem, exists in almost all the speed measuring radars. Due to it, the measured speeds of cars can sometimes be half or two times of the actual speeds. This problem gives wrong information to the police about the car speeds and may lead to serious consequences. Up to now, the main method to avoid this kind of error is to depend on human knowledge. However, this method can only reject some obviously wrong measured data and accuracy cannot be guaranteed. For example, if the measured speed of a fully loaded truck reaches up to 190 kilometers per hour, it can be easily proved wrong; while if the measured speed of a car is 200 kilometers per hour, it is hard to judge the correctness of this data according to human knowledge. Thus, the development of an effective frequency doubling suppression method is strongly needed which can increase the accuracy of the speed measuring and benefit a better traffic control.

\section{Working Principle of Speed Measuring Radars and Frequency Doubling Analysis}

Working Principle of Speed Measuring Radars. The working principle of speed measuring radars is based on Christian Johann Doppler theory. First, the fixed radar sends a series of radio waves which will be reflected by cars and generate echoes. Then the relative movement between the cars and the radar will change the frequencies of the echoes and the shifts of the frequencies will be proportionate to the relative speeds. This phenomenon is called Doppler Frequency Shift (DFS). Because the radar is fixed, the relative speeds equal the car speeds, which can be calculated from the frequency shifts. The topological structure of the speed measuring radars is shown in Fig. 1. 


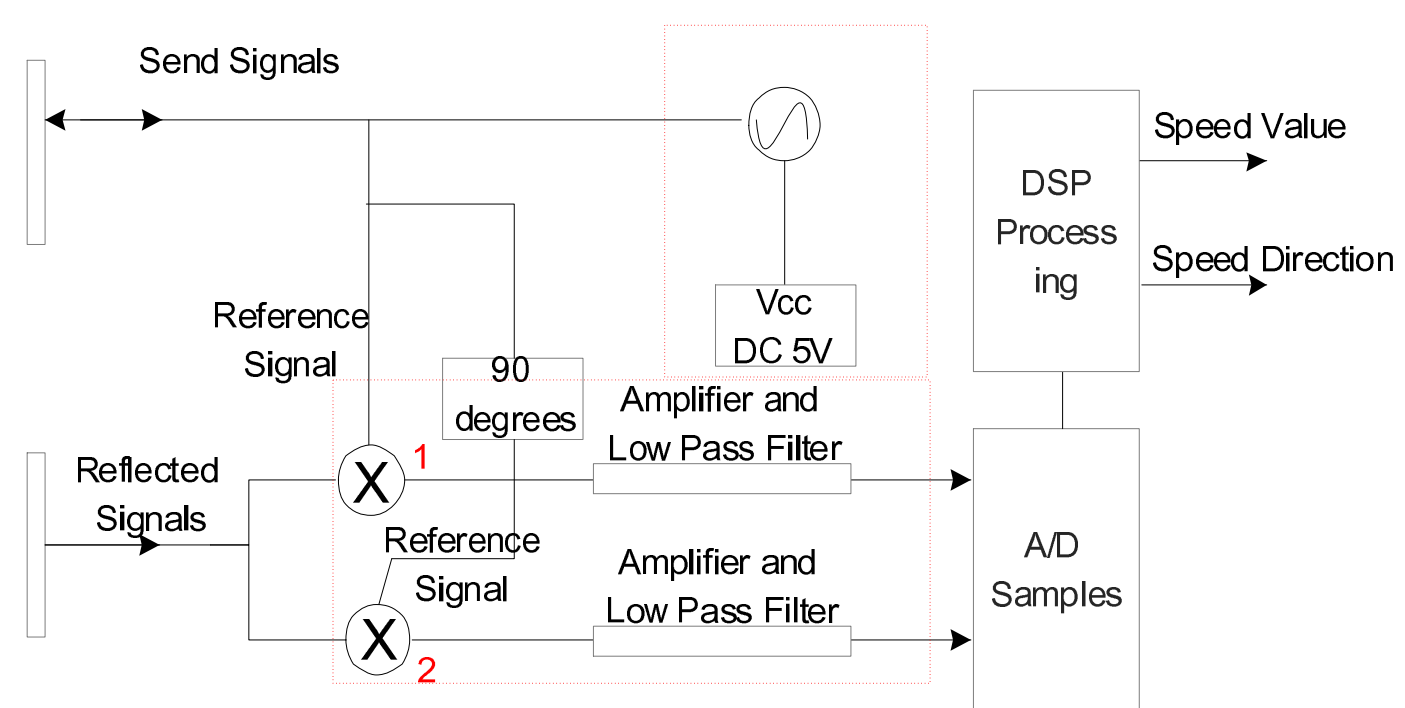

Fig. 1 Topological Structure of Speed Measuring Radars

As shown in Figure 1, the radio waves (RF) are generated by the Voltage Controlled Oscillator (VCO) and sent out through antennas. At the same time, the RF is divided into two parts, with one's phase shifted 90 degrees and the two parts are mixed with the received reflected waves. Based on this, the frequency shifts of the echoes which contain information about the car speeds can be extracted through a series of processing such as low pass filtration, amplification, A/D conversion and FFT calculation. This process can be expressed in math as the following.

The meanings of the notes are:

RF1: the reference signal generated by the VCO;

RF2: signal with 90 degrees shifts in phase;

$\mathrm{RC}$ : the reflected signal received by the radars;

$\mathrm{A}_{1}$ : gain of the reference signal;

$\mathrm{A}_{2}$ : gain of the phase-shifted signal;

$\mathrm{A}_{3}$ : gain of the reflected signal;

$\mathrm{A}_{4}$ : gain of the mixed signal in channel 1;

$\mathrm{A}_{5}$ : gain of the mixed signal in channel 2;

$\mathrm{f}_{0}$ : frequency of the reference signal;

$f_{d}$ : the frequency shift between the reference and the reflected signal;

$\varphi$ : initial phase of the reference signal;

$\varphi_{1}$ : phase of the mixed signals;

I: Mixed signal in channel 1;

Q: Mixed signal in channel 2;

v: car speeds;

$\mathrm{c}_{0}$ : light speed;

$\alpha$ : angle between the speed direction and the radar;

The reference signal generated by the VCO is:

$$
R F_{1}=A_{1} \cos \left(2 \pi f_{0} t+\varphi\right) .
$$

With phase shifted 90 degrees, the signal can be expressed as:

$$
R F_{2}=A_{2} \sin \left(2 \pi f_{0} t+\varphi\right)
$$


Then, the reflected signal is:

$$
R C=A_{3} \cos \left(2 \pi\left(f_{0} \pm f_{d}\right) t+\varphi\right) .
$$

The reference and reflected signals mix with each other and generate the mixed signals in channel 1 and 2 as:

$$
\begin{aligned}
& I=A_{4} \cos \left(2 \pi f_{d} t+\varphi_{1}\right) . \\
& Q=A_{5} \sin \left(2 \pi f_{d} t+\varphi_{1}\right) .
\end{aligned}
$$

Apply FFT transform to the two mixed signal $I$ and $Q$, information about the frequency shift $f_{d}$ can be extracted. Based on the relationship between the $f_{d}$ and the car speed :

$$
f_{d}=2 f_{0} \frac{v}{c_{0}} \cos \alpha,
$$

the car speed can be measured.

The above model describes the working principle of speed measuring radar for one car. However, in fact, more than one car will be detected and measured simultaneously, in which case multiple frequency shifts such as $\mathrm{f}_{\mathrm{d} 1}, \mathrm{f}_{\mathrm{d} 2}, \mathrm{f}_{\mathrm{d} 3}, \ldots, \mathrm{f}_{\mathrm{dn}}$ will be generated to calculate different car speeds.

Analysis of Frequency Doubling. According to the wave transmission characteristic of telecommunication, speed measuring radars can be taken as self-emitting and self-receiving wireless microwave communication terminals, while cars can be taken as a passive communication terminal due to the fact that moving cars simply reflect back the wireless microwave signal emitted by radars. In addition, differences between the distances, car shapes and materials will result in randomicity with the system gain and uncertainty with the passive communication channels. Thus, the whole system is a wireless communication random system in which the signal intensities decrease with the increase of the distances between radar and cars.

Neglecting the influence caused by the size of the reflecting plane of cars, the effective working distances of radars usually range from 0 to 50 meters. And the transmission loss of radar signal in free space can be expressed as:

$$
P_{L}=32.44+20 L g f+20 L g d \text {. }
$$

Where PL is the magnitude loss of the signal, $\mathrm{f}$ is the frequency of the signal and $\mathrm{d}$ is the distance to transmit.

Take $24.15 \mathrm{GHZ}$ speed measuring radars as an example. When the distance between a car and the fixed radar is 50 meters, the distance of wireless wave transmission is 100 meters and the magnitude decline of $24.15 \mathrm{GHZ}$ radar signal is $100.98 \mathrm{DB}$; while when the distance is only 1 meter, the distance of wave transmission is 2 meters and the magnitude decline is just 66.119DB. Therefore, when the car is at the furthest and nearest spots, due to the loss in electromagnetic wave space transmission, the intensities of signal differ by $34.86 \mathrm{DB}$.

Additionally, the actual environment is far more complex than the model above. Other effects such as different radar measuring angles, different reflecting intensity of cars and electromagnetic wave interruption will influence the magnitude of the transmission signal. Thus, the decline of the signal magnitude will be more than $34.86 \mathrm{DB}$. In this case, to guarantee that the radars can work effectively, high quality receiving circuit and signal processing method are needed, which can cope with such a large variation of signal amplitudes.

However, in order to realize sensitive detection of far and small cars, the present speed measuring radars mostly utilize high gain circuits, which directly leads to the fact that when some large cars or cars made of special materials are nearer to the radars, reflected signals will be over-intensified immediately and result in distortion. This kind of distortion signals will decrease the accuracy of the speed measuring. Fig. 2 shows the normal mixed signal $I$ and $Q$ and their FFT spectrum, while Fig. 3 
shows the distorted mixed signal $I, Q$ and their FFT spectrum. It can be seen that the distorted signals contain more multiple frequency signals than the normal ones, which will interfere with the correct measurement of the car speed.

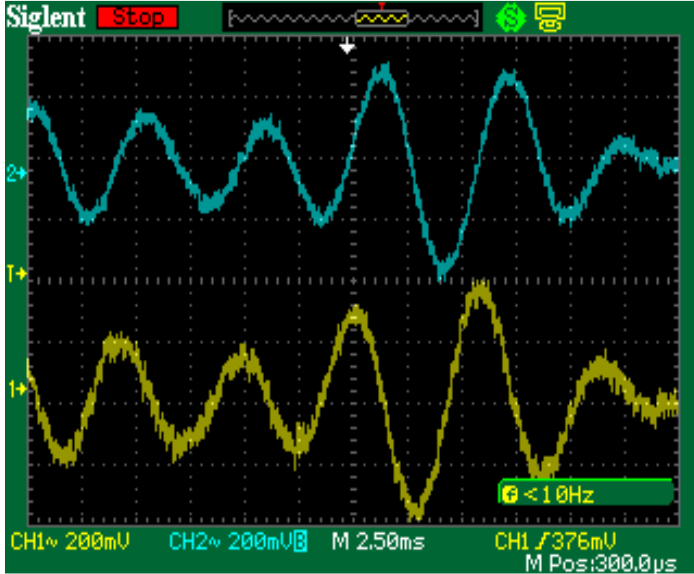

(a) Mixed Signals I and Q

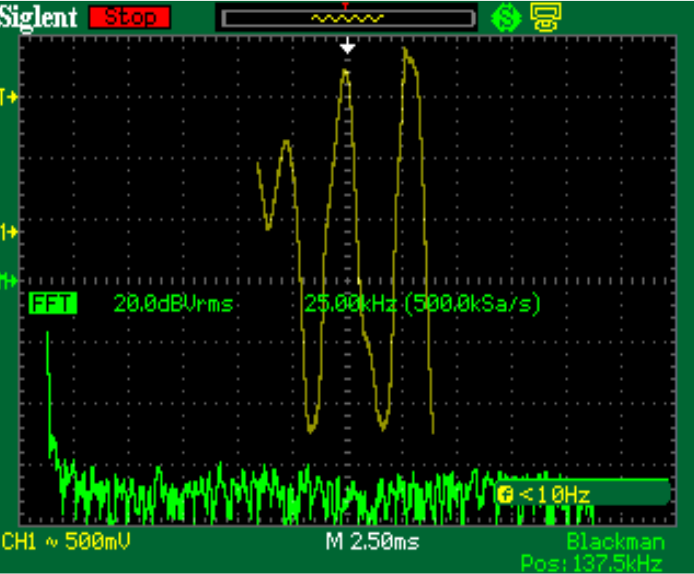

(b) Normal Signal FFT spectrum

Fig. 2 Normal Mixed Signals I (blue) and Q (yellow) and Their Signal FFT spectrum

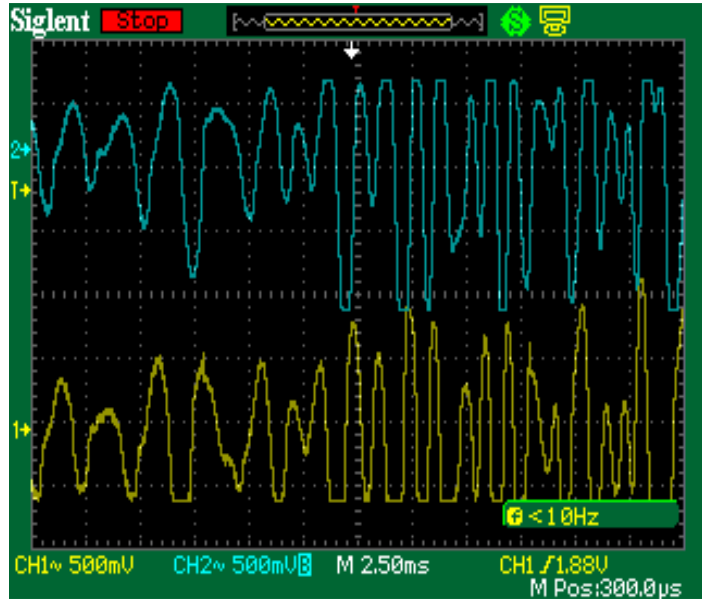

(a) Mixed Signals I and Q

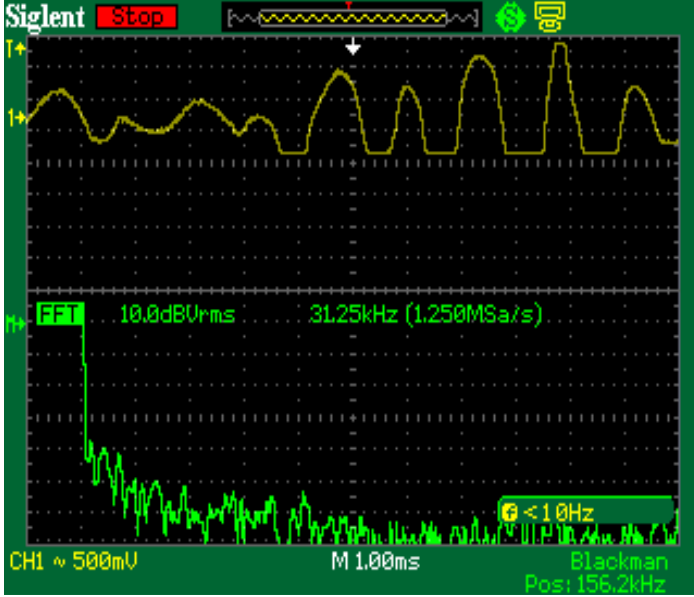

(b) Distorted Signal FFT spectrum

Fig.3 Distorted Mixed Signals I (blue) and Q (yellow) and Their Signal FFT spectrum

The distorted signal can be expressed as:

$I / Q=\sum_{I=1}^{N} A_{i} \bullet \cos \left(\omega_{I} \bullet t\right)$.

Multiple harmonic components are contained in the distorted signal and the half and doubling harmonic dominate. Therefore, the half and doubling harmonic will generate large components after FFT transformation, which are likely to be detected mistakenly as the car speeds.

In order to solve this problem, some radars introduce the method of matching detection of dominant wave and second harmonic. However, this method just enervates the effect of frequency doubling and cannot eliminate the interruption of speed measuring. Moreover, there are great possibilities to fail the examination of other cars with speed of twice or one-half of that of the proceeding one. Another method introduced by some radars is automatic gain control (AGC) module which can regulate the sensitivity of radars in order to make it compatible with both strong and weak signals. But the range that AGC can regulate is limited and working parameters settings are dependent on real working environments, which leads to the fact that it is complicated to operate and difficult to guarantee the optimal working state of radars.

Therefore, more effective distortion detection and evaluation system is needed to improve the accuracy of speed measuring radars. 


\section{Construction of Distortion Detection and Evaluation System Based on Digital Closed-loop Gain Automatic Control}

In order to solve the problem thoroughly, distortion detection and evaluation system and digital closed-loop gain automatic control technique are introduced to regulate and control the received signals of radars. The experiments and simulation prove that this method has effectively overcome the long-term existing problem of conventional speed measuring radars.

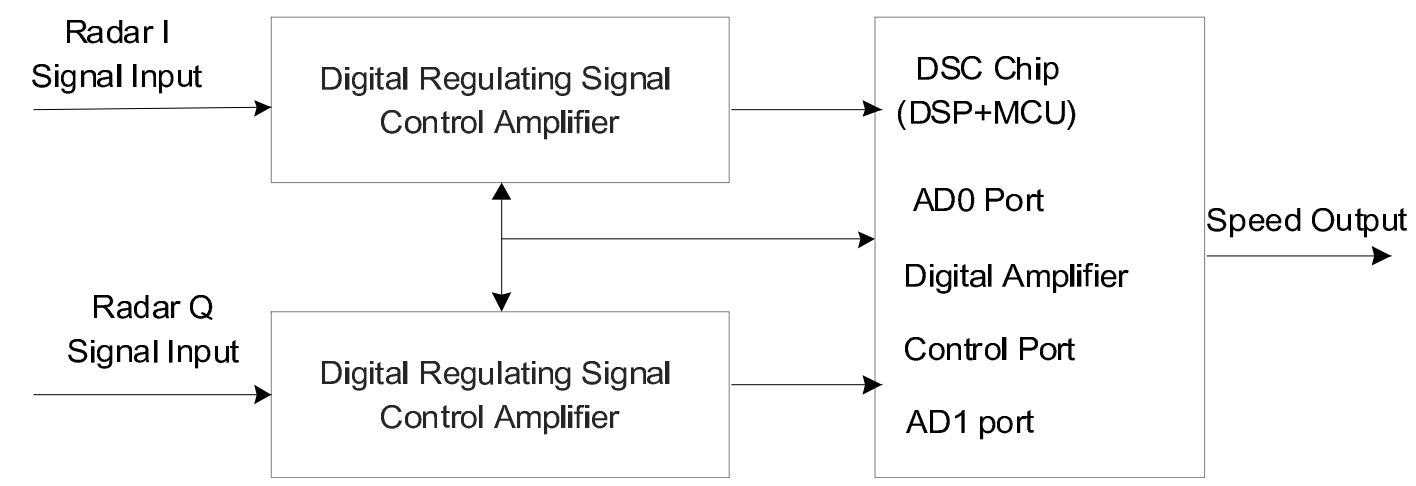

Fig. 4 Process of additional digital gain regulation and control between Doppler signal of radar output and DSP acquisition and processing

Specifically, one digital control closed-loop regulating circuit is introduced between the front-end input I/Q signal and terminal circuit with regulating range from -3DB 45DB up to 48DB. Based on the characteristic and formation of the signal clipping distortion, signal amplitude is firstly judged whether the signal is clipping distorted, after each $\mathrm{A} / \mathrm{D}$ acquisition of analog signals. FFT transformation of digital signal is conducted if distortion does not happen. If distortion occurs, reducing the gain of digital regulating gain amplifier by 3DB through the digital control port and analyze the acquisition again until the cease of distortion. The whole control utilizes the DSP software and each acquisition processing needs only $500 \mu \mathrm{s}$, without impact on the detection of cars and reducing the reaction time of radars.

\section{Conclusion}

In the research and development of speed measuring radar, this technique is successfully introduced to solve the problem which has puzzled the field of speed measuring radars. In the application of speed measuring radars, through the experiment and application of 10 settled speed measuring spot on high way for one year, none such as the phenomenon of frequency doubling has occurred. At the same time due to the application of the automatic amplification regulation of DSP program and sensitivity control, the use of speed measuring radars is very easy without complicated settings and debugging.

\section{References}

[1] Wang Xiaofei, Li Boquan, Pan Haibin: Design of signal processing system for Doppler speed radar based on SOPC. The 4th International Conference on Engineering Technologies and Ceeusro, 2011.

[2] Liu Zou: Data Sample and Processing of Speed Measuring Radar Based on DSP [D], Jiangnan University, 2008.

[3] Cem Unsal, Rahul Sukthankar and Chuck Thorpe: Functional Sensor Modeling for Automated Highway Systems simulations [C]. SPIE International Symposium on Intelligent Systems \& Advanced Manufacturing Technical Conference on Intelligent Transportation Systems, 1997. 
[4] Yang Chang-FA ,Ko Chuen-Jyi,Wu Boau-Cheng: A Free Space Approach for Extracting the equivalent Dielectric Constants of the Walls in Buildings,International Symposium on Antennas and Propagation Society,Digest,Volume:2,1996,Page(s):1036-1039

[5] Protat Alain, Williams, Christopher R: The Accuracy of Radar Estimates of Ice Terminal Fall Speed From Vertically Pointing Doppler Radar Measurements [J]. Journal of Applied Meteorology and Climatology, Vol. 50(10), 2011

[6] R. J. C. Bultitude, T. J. Willink, M. H. A. J. Herben and etc: Detection of Changes in the Spectra of Measured CW Mobile Radio Data for Space Wave Modelling Applications[J], 2007.

[7] B. Zhao and M. Valenti, "Some New Adaptive Protocols for the Wireless Relay Channel", Proc. Allerton Conf. Commun., Control, and Comp., 2003. 